\title{
Harvesting in an integrated general equilibrium model ${ }^{*}$
}

\author{
Thomas Eichner and Rüdiger Pethig \\ University of Siegen \\ Department of Economics \\ Hoelderlinstr. 3 \\ D-57068 Siegen \\ Germany \\ phone: ++49 (0) $2717403164 / 3143$ \\ fax: ++49(0) 2717402678 \\ e-mail: eichner@vwl.wiwi.uni-siegen.de \\ e-mail: pethig@vwl.wiwi.uni-siegen.de
}

\begin{abstract}
Harvesting of prey biomass is analyzed in an integrated ecological-economic system whose submodels, a predator-prey ecosystem and a simple economy, are microfounded dynamic general equilibrium models. These submodels are interdependent because the ecosystem responds to harvesting - through the reactions of optimizing individual organisms - by changing the provision of public ecosystem services to consumers. General analytical results are derived regarding the impact of harvesting policies on short-run equilibria of both submodels, on population dynamics, and on stationary states of the integrated model. A key insight is that prey biomass carries a positive ecosystem price which needs to be added as a tax mark-up to the economic price of harvested biomass to attain allocative efficiency. Further information on the dynamics is gained by resorting to numerical analysis of the policy regimes of zero harvesting, laissez-faire harvesting and efficient harvesting.

JEL classification: Q20, Q57

Key words: $\quad$ predator, prey, biomass price, harvesting
\end{abstract}

${ }^{*}$ Helpful comments from an anonymous referee are gratefully acknowledged. Remaining errors are the authors' sole responsibility. 
It "... is a matter of weighing costs and benefits of taking action, whether the action is the "inert" one of leaving resources alone in order to conserve them, or whether it involves exploiting a resource ... for so-called material ends".

David Pearce (1976, p. 320)

\section{Introduction}

There is a large literature on bioeconomic models of harvesting, e.g. Clark (1976), Ströbele and Wacker (1995), Eggert (1998) or Hoekstra and van den Bergh (2005), in which the ecosystem is modeled by a system of differential equations, one equation for every species, that describes the change in time of that species' population as a function of all species' populations. Based on the motivation and methods developed in particular, by Hannon (1976) and Crocker and Tschirhart (1992), in recent years an alternative approach has been put forward by Tschirhart (2000) and applied to harvesting of fish by Finnoff and Tschirhart (2003a, 2003b). Its innovative features are (i) the modeling of intra-ecosystem interdependence at the micro-level with representative organisms of all species exhibiting optimizing behavior and (ii) the coordination of all biomass transactions in the ecosystem through a general equilibrium allocation mechanism. These basic building blocks are also used by Christiaans et al. (2006) in their approach to the ecosystem. But while Tschirhart and Finnoff equilibrate the individual organisms' transactions through endogenous transactions costs, Christiaans et al. treat individual organisms like economic consumers and apply the competitive general equilibrium mechanism to coordinate transactions in the ecosystem.

The present paper takes up Christiaans et al. (2006)'s approach for modeling a simple ecosystem in predator-prey relationship where a prey species is 'preyed upon' by a predator species and by humans. The ecosystem is linked to a simple general equilibrium model of the economy that depends on ecosystem services which are increasing in species densities. In other words, the ecosystem and the economy are interdependent submodels of an integrated ecological-economic system (IEES). Each submodel is operated by an allocation mechanism of its own that determines the adaptions and feedback effects of one submodel to changes or shocks originating from the other submodel. Thus we aim to analyze harvesting in a new approach to ecological interdependence and to demonstrate the strengths and new insights of that approach as compared to conventional models. Humans and animal predators compete for prey biomass where humans are restrained only by their resources and selfinterest. A key insight is that prey biomass carries a positive price in the ecosystem while 
on competitive markets of the unregulated economy that biomass would be considered a free good and hence be sold at marginal harvesting cost. Efficiency is shown to require a harvesting tax equal to the ecosystem price of biomass.

Given the fairly complex setup of the IEES model and our aim to provide a transparent exposition of its analytical structure and solution we resort to simplifying assumptions especially on the harvesting technology and on consumer preferences that somewhat reduce but not eliminate the interdependencies of the submodels. As a reward, important results on short-run equilibria, on population dynamics and on stationary states of the IEES can be derived analytically. More concrete insights on the time path of the system toward its stationary state are gained by resorting to numerical analysis. The simulations allow to trace the changes in time of all variables including all prices in the ecosystem and in the economy as well as the efficient harvest and the efficient rate of the harvesting tax.

In Section 2 the building blocks of the IEES model are introduced. Section 3.1 explores the short-run equilibria in the economy and in the ecosystem taking as given the populations of species, the amount of harvested biomass and the rate of the harvesting tax. Section 3.2 presents analytical results on the population dynamics and stationary states for predetermined harvesting tax policies and also characterizes the efficient tax policy. Numerical analysis of the ecological-economic dynamics follow in Section 4 where harvesting scenarios are investigated in a sequence: zero-harvesting, laissez-faire harvesting and efficient harvesting.

\section{The building blocks of the model}

Consider a simple economy producing the amount $y$ of a consumer good by means of the linear production function

$$
y=\frac{\ell_{y}}{c_{y}}
$$

where $\ell_{y}$ denotes the labor input and $c_{y}>0$ is an input-output coefficient.

Humans are top predators and compete with the predator species in the ecosystem for prey biomass. Let $h$ be the biomass of the prey species harvested by humans. The harvesting technology takes the form

$$
h=\frac{\ell_{h}}{c_{h}}
$$

where the amount of labor, $\ell_{h}$, is the harvesting effort and $c_{h}>0$ is an input-output coefficient. We concede that the harvesting function (2) is oversimplified as it excludes, in 
particular, the empirically relevant positive stock externality of an increasing prey population that rightly is considered in the state-of-the-arts fisheries literature. We only defend (2) on the grounds that it allows for a transparent exposition of the complex model yielding, at the same time, informative analytical results. In a much more general setup, Eichner and Pethig (2006c) employ a harvesting function with such a stock externality, and they elaborate its significant impact on ecosystem prices.

The output $y$ and the harvest $h$ are private (and therefore marketable) consumer goods. In addition, consumers enjoy public (and therefore non-marketable) ecosystem services that are assumed to be positively correlated to the species' populations. To simplify, all consumers are supposed to be identical. The representative consumer's preferences are given by the utility function

$$
u=y^{\kappa} h^{1-\kappa}+\varepsilon n_{2} \quad(\kappa, \varepsilon>0) .
$$

In (3), the preferences for $y$ and $h$ are Cobb-Douglas with $\kappa$ reflecting the preference weight of product $y$. The public ecosystem service is assumed to be proportional to the population of species $2, n_{2}$, and it does not depend on $n_{1}$. The size of the preference parameter $\varepsilon$ indicates how essential this ecosystem service is for consumers. The simplifying assumption of the utility function being additively separable in the ecosystem service prevents changes in the provision of ecosystem services from causing repercussions in the economy which in turn would affect the ecosystem through changes in harvesting. ${ }^{1}$

The economy submodel is completed by the labor constraint

$$
\bar{\ell}=\ell_{y}+\ell_{h}
$$

where the aggregate labor supply $\bar{\ell}$ is exogenously given.

The ecosystem is made up of a microfounded predator-prey model where the predator (species 2) feeds on a prey species (species 1) and the prey species 'feeds' on an exogenously given resource $\bar{r}$. Basic units of analysis are a representative individual organism of species 1 and a representative individual organism of species 2. These organisms generate net offspring according to the functions

$$
\begin{aligned}
& b_{1}=\left(r_{1}\right)^{\phi_{1}}\left(\bar{z}_{1}-z_{1}\right)^{\psi_{1}}-\gamma_{1}, \\
& b_{2}=\left(z_{2}\right)^{\phi_{2}}-\gamma_{2},
\end{aligned}
$$

respectively. In (5), $r_{1}$ denotes the prey's intake of the resource, $z_{2}$ denotes the predator's intake of prey biomass and $z_{1}$ denotes the prey's loss of own biomass to its predators. $z_{1}$ is

\footnotetext{
${ }^{1}$ These consequences of (3) are also pointed out in Section 3.1 (below equations (11)) and in Section 4.2 (footnote 18). For the important consequences in assuming separability between the market and nonmarket goods, more generally, see also Deaton (1981).
} 
bounded from above by $\bar{z}_{1}$, a positive constant. $\gamma_{1}$ and $\gamma_{2}$ are positive constant mortality rates and $\phi_{1}, \phi_{2}$ and $\psi_{1}$ are positive parameters reflecting the elasticity of the net offspring generation with respect to prey biomass intake and loss of own biomass, respectively. ${ }^{2}$ With $n_{i}$ denoting the population of species $i$ the population growth of species $i$ is given by the differential equations

$$
\frac{\mathrm{d} n_{i}}{\mathrm{~d} t}=\dot{n}_{i}=n_{i} b_{i} \quad i=1,2 .
$$

The ecosystem submodel is closed by the resource constraints

$$
\begin{aligned}
\bar{r} & =n_{1} r_{1}, \\
n_{1} z_{1} & =h+n_{2} z_{2} .
\end{aligned}
$$

Equation (7) [(8)] equilibrates the demand for and the supply of the resource [prey biomass].

\section{General equilibrium and corrective taxation in the in- tegrated system}

\subsection{Short-run equilibria in both submodels}

We now introduce competitive markets in both the economy and the ecosystem. Along conventional lines, the market economy exhibits a competitive market for labor with price $p_{\ell}$, a market for the produced good with price $p_{y}$ and a market for the prey biomass harvested with price $p_{h}$. These markets are in operation at each point in time. In addition to competitive prices we introduce a tax on harvested biomass, $\tau$, levied on the harvesting firm. The producer and the harvester maximize profits as price takers and the consumer maximizes her utility subject to her budget constraint. The associated optimization problems are ${ }^{3}$

$$
\begin{array}{ll}
\max _{\ell_{y}} & p_{y} \frac{\ell_{y}}{c_{y}}-p_{\ell} \ell_{y}, \\
\max _{\ell_{h}} & \left(p_{h}-\tau\right) \frac{\ell_{h}}{c_{h}}-p_{\ell} \ell_{h}, \\
\max _{y, h} & y^{\kappa} h^{1-\kappa}+\varepsilon n_{2}+\rho_{c}\left(p_{\ell} \bar{\ell}+w-p_{y} y-p_{h} h\right),
\end{array}
$$

\footnotetext{
${ }^{2}$ The basic hypothesis that net offspring is negatively correlated with the loss of own biomass to predators, the mortality rate and reduced food uptake is supported in much of the biological literature (e.g. Stephens and Krebs (1986)). See also the similar hypotheses applied by Tschirhart (2000) and Finnoff and Tschirhart (2003a, 2003b). The functional form Cobb-Douglas only serves to make the analysis tractable. See also footnote 10.

${ }^{3}$ All agents are modeled as myopic optimizers. Assuming infinitely lived agents, instead, who maximize the present value of their instantaneous objective function would not change the results.
} 
where $\rho_{c}$ is a Lagrange multiplier and $w:=\tau h$ is the tax revenues transferred lumpsum to the consumer. Solving $(9 a)-(9 c)$ we obtain the first-order conditions

$$
p_{y}=c_{y} p_{\ell}, \quad p_{h}-\tau=c_{h} p_{\ell} \quad \text { and } \quad \frac{\kappa h}{(1-\kappa) y}=\frac{p_{y}}{p_{h}} .
$$

For any given tax rate $\tau$ and any ecosystem service $n_{2}$ the equilibrium prices $\left(p_{y}, p_{h}, p_{\ell}\right)$ and the economic equilibrium allocation $\left(h, y, \ell_{h}, \ell_{y}\right)$ need to be determined by $(1),(2),(4)$ and (10). Closer inspection shows that there are seven variables and six equations. To eliminate the degree of freedom we choose labor as the numeraire good, $p_{\ell} \equiv 1$. Solving the equations (1), (2), (4) and (10) then yields

$$
\begin{aligned}
p_{y} & =c_{y}, \\
p_{h} & =c_{h}+\tau, \\
h & =\frac{\bar{\ell}(1-\kappa)}{c_{h}+\kappa \tau c_{y}}, \\
y & =\frac{\bar{\ell} \kappa\left(c_{h}+\tau\right)}{c_{y}\left(c_{h}+\kappa \tau\right)}, \\
\ell_{h} & =\frac{c_{h} \bar{\ell}(1-\kappa)}{c_{h}+\kappa \tau c_{y}}, \\
\ell_{y} & =\frac{\bar{\ell} \kappa\left(c_{h}+\tau\right)}{c_{h}+\kappa \tau} .
\end{aligned}
$$

Note that the short-run competitive equilibrium (11) of the economy submodel is contingent on the harvesting tax rate $\tau$. In a more general approach it would also depend on the level of public ecosystem services which we have set equal to $n_{2}$. That dependence is absent in our simple parametric model because $n_{2}$ enters the utility function (3) as an additive term.

In the ecosystem submodel, at each point in time there is a competitive market for the resource with price $\pi_{r}$ and for prey biomass with price $\pi_{z}$. All prices are denominated in virtual units of account. In fact, non-human species definitively do not use money while modern economies definitely do rely on money. Yet it is interesting to observe that the standard neoclassical general equilibrium model of the Debreu type does not describe an economy with real (fiat or commodity) money. That theory is readily and consistently interpreted (i) as dealing with assigning shadow prices (or scarcities) to resources, (ii) as considering rational agents perceiving these scarcities and coping with them under appropriate constraints and (iii) as envisaging an equilibrium state of 'market clearing' that essentially expresses the idea of an allocation process reaching a state in which all agents' perceptions of scarcities are consistent. It is exactly this type of allocation mechanism that we introduce into the ecosystem and that operates independent of the competitive mechanism in the economy. ${ }^{4}$

\footnotetext{
${ }^{4}$ For an ecosystem in isolation this approach has gradually been developed, with varying hypotheses and
} 
In the short run, all populations are fixed. Following Christiaans et al. (2006) we assume that the representative organisms behave as if they maximize their net offspring subject to the constraint

$$
\begin{aligned}
\pi_{r} \omega_{1}+\pi_{z}\left(z_{1}-\frac{h}{n_{1}}\right) & =\pi_{r} r_{1} \\
\pi_{r} \omega_{2} & =\pi_{z} z_{2}
\end{aligned}
$$

respectively. $\omega_{i}$ is organism $i$ 's 'resource endowment' defined as

$$
\omega_{i}:=\frac{\sigma_{i} \bar{r}}{\sigma_{1} n_{1}+\sigma_{2} n_{2}},
$$

where $\sigma_{i} \geq 0$ is a species-specific parameter. The transactions constraints (12) closely resemble budget constraints of economic consumers where $\pi_{r} \omega_{i}$ (for $i=1,2$ ) represent exogenous incomes and where $\pi_{z} z_{1}$ is the analogue of labor income. Although these transactions constraints appear to be a mechanical export of conventional economics at first glance, they are in fact an appropriate description of preying or feeding opportunities of nonhuman species in the ecosystem. To show that consider first the exogenous income $\pi_{r} \omega_{i}$ (setting $z_{1}=h=0$ in equation (12a) for the time being). In any given short-run period organism $i$ is able to take up the amount

$$
r_{1}=\frac{\sigma_{1} \bar{r}}{\sum_{j} \sigma_{j} n_{j}} \quad \text { or } \quad z_{2}=\frac{\pi_{r} \sigma_{2} \bar{r}}{\pi_{z} \sum_{j} \sigma_{j} n_{j}}
$$

of food (prey biomass) without being forced to sacrifice own biomass. Ceteris paribus, the amount $z_{2}$ from (14) is the smaller,

(i) the more abundant is the resource $\left(\pi_{r}\right.$ small),

(ii) the scarcer is the prey biomass $\left(\pi_{z}\right.$ large),

(iii) the more populated is the ecosystem $\left(n_{1}\right.$ and $n_{2}$ large),

(iv) the greater is $\sigma_{1}$.

Note that $\pi_{r}, \pi_{z}, n_{1}$ and $n_{2}$ are time-specific while $\sigma_{1}$ and $\sigma_{2}$ are time-invariant parameters. Other things being equal $\sigma_{i}$ determines organism $i$ 's 'purchasing power' for risk free shopping! It is therefore plausible to assume that $\sigma_{i}$ is the greater, the higher is species $i$ 's trophical level, i.e. its status or 'power' as a predator. Moreover, $\omega_{i}$ from (13) can be interpreted as organism $i$ 's ownership share of the resource $\bar{r}$, since $\sum_{j} n_{j} \omega_{j}=\bar{r}$. The shares change over time as long as one or more populations are not stationary. From a more technical general equilibrium point of view the resource stock $\bar{r}$ represents a valuable asset endowments of which need to be assigned to all organisms to keep the ecosystem model in different versions, by Eichner and Pethig (2003, 2006a, 2006b) and Christiaans et al. (2006). 
closed. ${ }^{5}$ Note, however, that in contrast to the wealth distribution among economic agents, in the ecosystem ownership shares cannot be arbitrarily assigned since the $\sigma_{i}$ are specific genetic parameters reflecting the organisms' predation power.

Suppose next (temporarily) that $\omega_{1}=h=0$ in equation (12a) such that the pertaining transactions constraint of organism 1 reads

$$
z_{1}=\frac{\pi_{r}}{\pi_{z}} r_{1}
$$

Excluding an exogenous income and the loss of own biomass to humans, (15) conveys the information to organism 1 that if it wants to consume the amount $r_{1}$ of the resource it is bound to sacrifice $\left(\pi_{r} / \pi_{z}\right) r_{1}$ of own biomass to its predators because it exposes itself to its predators while foraging. Such a risk of being preyed upon while preying is known as the predation risk in the ecological literature (e.g. Lima and Dik (1990)) and motivates (15). ${ }^{6}$ According to (15) the sacrifice of own biomass necessary to consume a given amount of the resource is the greater,

(i) the scarcer is the resource $\left(\pi_{r}\right.$ large)

(ii) and the more abundant is organism 1's own biomass $\left(\pi_{z}\right.$ small).

Hence the predation risk in not constant over time as long as no stationary state is attained.

Consider now (12a) with $\omega_{1}>0$ and $h=0$, yielding $\pi_{r} \omega_{1}+\pi_{z} z_{1}=\pi_{r} r_{1}$. In this case organism $i$ can obviously choose to content itself with risk free foraging by choosing $z_{1}=0$ and restricting $r_{1}$ to $\omega_{1}$. If it prefers to consume $r_{1}>\omega_{1}$ it needs to expose itself to some predation risk and attains $r_{1}-\omega_{1}>0$ extra food only by sacrificing the amount $\pi_{z} z_{1} / \pi_{r}$ of own biomass. Observe that the top predator species 2 is not exposed to any predation risk. It cannot earn a second income from selling own biomass because its biomass is zero priced due to the absence of any demand for it. Nevertheless, owing to organism 2's higher trophical level, its exogenous income $\pi_{r} \omega_{2}$ may well exceed organism 1's total income as determined by the RHS of equation (12a).

Suppose finally $h>0$. Clearly, since $z_{1}$ is defined as organism $i$ 's aggregate loss of own biomass to humans and to the predator species 2 (see $(5 \mathrm{a})) z_{1} \geq\left(h / n_{1}\right)$ is required for feasibility. Moreover, the amount $\pi_{z} h / n_{1}$ of its own biomass is lost to the humans without

\footnotetext{
${ }^{5}$ In earlier papers of ours, e.g. Christiaans et al. (2006), organism $i$ had been assigned a time-invariant lumpsum income $e_{i}$ (in units of account) instead of the exogenous income $\pi_{r} \omega_{i}$ used in the present approach. The distinction between a physical endowment $\omega_{i}$ and its value $\pi_{r} \omega_{i}$ both of which may vary over time is necessary to establish a complete (neoclassical) general equilibrium model of the ecosystem.

${ }^{6}$ With the same motivation, Tschirhart (2000) assumes that the organism's supply of own biomass depends on (nothing but) the demand for prey biomass. In Tschirhart's parametric model the equivalent of (15) reads $z_{1}=\delta r_{1}^{\alpha}$, where the $\delta$ and $\alpha$ are positive constants.
} 
compensation. Hence for given $z_{1}$ organism 1's resource intake is the smaller, the greater is $h$.

Solving the organisms' optimization problems

$$
\begin{array}{ll}
\max _{r_{1}, z_{1}} & \left(r_{1}\right)^{\phi_{1}}\left(\bar{z}_{1}-z_{1}\right)^{\psi_{1}}-\gamma_{1}+\rho_{1}\left[\pi_{r}\left(\omega_{1}-r_{1}\right)+\pi_{z}\left(z_{1}-\frac{h}{n_{1}}\right)\right], \\
\max _{z_{2}} & \left(z_{2}\right)^{\phi_{2}}-\gamma_{2}+\rho_{2}\left(\pi_{r} \omega_{2}-\pi_{z} z_{2}\right)
\end{array}
$$

yields the first-order condition

$$
\frac{\phi_{1}\left(\bar{z}_{1}-z_{1}\right)}{\psi_{1} r_{1}}=\frac{\pi_{r}}{\pi_{z}}
$$

After some rearrangement of terms we obtain the demand and supply functions

$$
\begin{aligned}
r_{1} & =\frac{\pi_{z} \phi_{1}\left(\bar{z}_{1}-\frac{h}{n_{1}}\right)+\phi_{1} \pi_{r} \omega_{1}}{\pi_{r}\left(\phi_{1}+\psi_{1}\right)}, \\
z_{1} & =\frac{\pi_{z}\left(\phi_{1} \bar{z}_{1}+\psi_{1} \frac{h}{n_{1}}\right)-\psi_{1} \pi_{r} \omega_{1}}{\pi_{z}\left(\phi_{1}+\psi_{1}\right)}, \\
z_{2} & =\frac{\pi_{r} \omega_{2}}{\pi_{z}} .
\end{aligned}
$$

Taking Walras' law into account, we observe that for any given harvesting activity $h$ there are five variables, namely the equilibrium ecosystem prices $\left(\pi_{r}, \pi_{z}\right)$ and the equilibrium ecosystem allocation $\left(r_{1}, z_{1}, z_{2}\right)$ that need to satisfy the four equations (8) and (18a)-(18c). Hence there exists a degree of freedom which we eliminate by choosing the resource as the numeraire good, ${ }^{7} \pi_{r} \equiv 1$. With this convention we calculate the equilibrium ecosystem price of biomass of species 1 as

$$
\pi_{z}=\frac{n_{1} \psi_{1}\left(\frac{\sigma_{1} \bar{r}}{\sigma_{1} n_{1}+\sigma_{2} n_{2}}\right)+n_{2}\left(\phi_{1}+\psi_{1}\right)\left(\frac{\sigma_{2} \bar{r}}{\sigma_{1} n_{1}+\sigma_{2} n_{2}}\right)}{n_{1} \phi_{1}\left(\bar{z}_{1}-\frac{h}{n_{1}}\right)}
$$

and the equilibrium demands and supplies as

$$
\begin{aligned}
r_{1} & =\frac{\bar{r}}{n_{1}} \\
z_{1} & =\frac{\left(n_{1} \sigma_{1}+n_{2} \sigma_{2}\right) \psi_{1} \frac{h}{n_{1}}+n_{2} \sigma_{2} \phi_{1} \bar{z}_{1}}{n_{1} \sigma_{1} \psi_{1}+n_{2} \sigma_{2}\left(\phi_{1}+\psi_{1}\right)}, \\
z_{2} & =\frac{n_{1} \sigma_{2} \phi_{1}\left(\bar{z}_{1}-\frac{h}{n_{1}}\right)}{n_{1} \sigma_{1} \psi_{1}+n_{2} \sigma_{2}\left(\phi_{1}+\psi_{1}\right)} .
\end{aligned}
$$

\footnotetext{
${ }^{7}$ Since prey biomass is the only commodity that is traded in both submodels it would be near at hand to choose biomass as numeraire in both submodels. The only reason for not proceeding along that line is analytical convenience.
} 
According to (19) and (20), the short-run competitive ecosystem equilibrium does not only depend on the population levels $n_{1}$ and $n_{2}$, but also on the amount of biomass harvested which makes the ecosystem submodel dependent on the economy submodel. We readily infer from (19) that the ecosystem price of biomass rises when humans increase their harvest of biomass, $h$.

Summing up the preceding analysis, we showed that for each point in time the economic equilibrium is contingent on $n_{2}$ whereas the instantaneous ecosystem equilibrium is contingent on $h$. Moreover, as is evident from the equations (11d) - (11f), the tax rate $\tau$ not only has an impact on the resource allocation in the economy but it also affects the allocation in the ecosystem through determining the harvest of biomass in (11c). The tax therefore turns out to be a powerful instrument for nature conservation. Once $h$ is specified for each short-run period, the transactions (20) are fully determined.

\subsection{The harvesting tax, population dynamics and ecological sta- tionary states}

Inserting (20) into (5) and (6) yields the population growth functions

$$
\begin{aligned}
& \dot{n}_{1}=n_{1} \cdot\left[\left(\frac{\bar{r}}{n_{1}}\right)^{\phi_{1}} \cdot\left(\frac{\left(n_{1} \sigma_{1}+n_{2} \sigma_{2}\right) \psi_{1}\left(\bar{z}_{1}-\frac{h}{n_{1}}\right)}{n_{1} \sigma_{1} \psi_{1}+n_{2} \sigma_{2}\left(\phi_{1}+\psi_{1}\right)}\right)^{\psi_{1}}-\gamma_{1}\right], \\
& \dot{n}_{2}=n_{2} \cdot\left[\left(\frac{n_{1} \phi_{1} \sigma_{2}\left(\bar{z}_{1}-\frac{h}{n_{1}}\right)}{n_{1} \sigma_{1} \psi_{1}+n_{2} \sigma_{2}\left(\phi_{1}+\psi_{1}\right)}\right)^{\phi_{2}}-\gamma_{2}\right] .
\end{aligned}
$$

The differential equations (21) fully describe the population dynamics for any given time path of $h$ and any initial populations $n_{1}(0)$ and $n_{2}(0)$. Note that $(21)$ is derived from the ecosystem submodel of the present paper while the conventional bioeconomic models of harvesting cited in the introduction employ predator-prey models that are macro approaches starting out with a pair of (parametric) differential equations of the type $n_{i}=N^{i}\left(n_{1}, n_{2}\right)$ for $i=1,2$. Harvesting of species 1 is accounted for in these kinds of models (see e.g. Ströbele and Wacker 1995, p. 70) by simply subtracting $h$ from $n_{1}=N^{1}\left(n_{1}, n_{2}\right)$, i.e. by substituting $n_{1}=N^{1}\left(n_{1}, n_{2}\right)-h$ for $n_{1}=N^{1}\left(n_{1}, n_{2}\right)$. In contrast, the differential equations (21) take the form $n_{i}=N^{i}\left(n_{1}, n_{2}, h\right)$ with $N_{h}^{i}<0$ for $i=1,2$. Table 1 further details the differential treatment of harvesting in conventional macro approaches and the present approach to population dynamics. 


\begin{tabular}{|c|c|c|c|c|c|}
\hline & & $\frac{\partial \dot{n}_{i}}{\partial h_{i}}$ & & $\frac{\partial^{2} \dot{n}_{i}}{\partial h_{i}^{2}}$ & $\begin{array}{c}\frac{\partial \dot{n}_{i}}{\partial h_{i} \partial n_{j}} \\
(i=j \text { or } i \neq j)\end{array}$ \\
\hline \multirow{2}{*}{$\begin{array}{l}\text { conventional } \\
\text { approaches }\end{array}$} & $i=1$ & -1 & & 0 & 0 \\
\hline & $i=2$ & 0 & & 0 & 0 \\
\hline \multirow{2}{*}{$\begin{array}{l}\text { present } \\
\text { approach }\end{array}$} & $i=1$ & $<0$ & $\gtreqless 0$ & $\Longleftrightarrow \quad \psi_{1} \gtreqless 1$ & $\neq 0$ \\
\hline & $i=2$ & $<0$ & $\gtreqless$ & $\Longleftrightarrow \quad \psi_{2} \gtreqless 1$ & $\neq 0$ \\
\hline
\end{tabular}

Table 1: Alternative ways of modeling predator-prey systems with harvesting

Recall that the growth functions (21) specify the population dynamics only for predetermined time paths of $h$ which, in turn, depend on time paths of $\tau$ via (11c). In fact, defining $H(\tau):=\frac{\bar{\ell}(1-\kappa)}{c_{h}+\kappa \tau c_{y}}$ we find that $H_{\tau}<0, H(0)=\bar{\ell}(1-\kappa) / c_{h}$ and $H(\tau) \rightarrow 0$ for $\tau \rightarrow \infty$. Due to the strict monotonicity of the function $H$ it is equivalent to take as the relevant policy instrument either the harvesting tax or the harvesting quota, $h$. We proceed by focusing on harvesting tax policies.

Although the efficient intertemporal taxation of harvesting is of prime interest we postpone that issue to first explore some general properties of the IEES equilibrium in the very long run under the

Assumption 1. The exogenously given tax policy $\{\tau(t)\}$ satisfies $\tau(t) \in[0, \infty[$ for all $t \geq 0$, and there is $\tilde{t} \geq 0$ such that $\tau(t)=\tau$ for all $t \geq \tilde{t}$.

The impact of such a tax policy on the stationary state of (21) and its stability features are specified in

\section{Proposition 1.}

Let $\{\tau(t)\}$ be a tax policy satisfying the Assumption 1. Suppose $e^{8}$ there are functions $F^{i}$ : $\mathbb{R}_{+} \longrightarrow \mathbb{R}_{+}$such that $n_{i}=F^{i}(\tau)$ and $\left[F^{1}(\tau), F^{2}(\tau)\right]$ is the unique stationary state of (21) and suppose the constraint $M(\tau)>0$ defined in the Appendix is satisfied. Then the stationary state is locally asymptotically stable.

The message of Proposition 1 is that there exists a range of parameters and tax rates for which the IEES runs into its unique stationary state (although it is not clear how restrictive the constraint $M(\tau)>0$ is).

Having established sufficient conditions for local asymptotic stability of the stationary state we are now in the position to investigate the long-run allocative effects of tax rate changes. To be more specific, assume that Assumption 1 holds and take the associated

\footnotetext{
${ }^{8}$ The existence of such functions is supported by the authors' numerical analysis.
} 
stationary state $\left[F^{1}(\tau), F^{2}(\tau)\right]$ as the starting point of a comparative dynamic exercise. The results are reported in

\section{Proposition 2.}

Let $\{\tau(t)\}$ be a tax policy satisfying the Assumption 1 and suppose the associated stationary state of the IEES is disturbed by a small increase in the tax rate for all $t \geq \tilde{t}$. The long-run displacement effects of this shock are listed in Table 2 for the economic submodel and in Table 3 for the ecosystem submodel.

\begin{tabular}{|c||c|c|c|c|c|c|c|c|}
\hline & $\mathrm{d} p_{y}$ & $\mathrm{~d} p_{h}$ & $\mathrm{~d} p_{\ell}$ & $\mathrm{d} h$ & $\mathrm{~d} \ell_{h}$ & $\mathrm{~d} y$ & $\mathrm{~d} \ell_{y}$ & $\mathrm{~d} u$ \\
\hline \hline $\mathrm{d} \tau>0$ & 0 & + & $\equiv 0$ & - & - & + & + & $?$ \\
\hline
\end{tabular}

Table 2: The impact of a tax increase on the stationary state of the economy

\begin{tabular}{|c||c|c|c|c|c|c|c|c|c|}
\hline & $\mathrm{d} n_{1}$ & $\mathrm{~d} n_{2}$ & $\mathrm{~d} \omega_{1}$ & $\mathrm{~d} \omega_{2}$ & $\mathrm{~d} r_{1}$ & $\mathrm{~d} z_{1}$ & $\mathrm{~d} z_{2}$ & $\mathrm{~d} \pi_{r}$ & $\mathrm{~d} \pi_{z}$ \\
\hline \hline $\mathrm{d} \tau>0$ & + & + & - & - & - & - & 0 & $\equiv 0$ & - \\
\hline
\end{tabular}

Table 3: The impact of a tax increase on the stationary state of the ecosystem

Proposition 2 demonstrates the explanatory power of the present approach in tracing long-run effects on prices and quantities not only in the economy but also in the ecosystem. The impact of the tax hike on the economy is straightforward: since biomass becomes more expensive, labor is shifted from harvesting to production of the consumer good. ${ }^{9}$ The net effect of these changes on the representative consumer's utility is not clear. She consumes less biomass, a greater amount of the consumer good and more ecosystem services because $\mathrm{d} n_{2} / \mathrm{d} \tau>0$ (as shown in Table 3). The reduction in the amount of biomass harvested has important consequences for the long-run allocation in the ecosystem. The long-run populations of both species increase (which we proved in the Appendix by showing that $F_{\tau}^{i}>0$ for $\left.i=1,2\right)$. Thus the competition among all organisms is intensified resulting in diminished endowments (13). Since prey organisms now must 'sell' their own biomass at a lower price $\left(\mathrm{d} \pi_{z}<0\right)$ they reduce their respective supply. With lower biomass and resource incomes they are forced to content themselves with less resource intake. The predators lose income but are able to uphold their consumption of prey biomass nonetheless because prey biomass has become less expensive. ${ }^{10}$

\footnotetext{
${ }^{9}$ The price $p_{y}$ remains unchanged due to the linear production technology.

${ }^{10}$ This special effect is a consequence of the Cobb-Douglas specification of the net offspring functions (5a) and (5b). Cobb-Douglas is used here primarily for convenience of exposition. More complex and flexible functional forms as e.g. CES functions may be more appropriate but they do not lend themselves easily to
} 
In (21) as well as in Proposition 2 we focused on predetermined harvesting strategies satisfying Assumption 1 and on small exogenous changes in those strategies without assessing their efficiency. To address the efficiency issue, we could follow here the conventional approach of bioeconomic harvesting models in the literature by envisaging a social planner who seeks to maximize the present value of the representative consumer's utility (3) subject to (1), (2), (4) and (21). Yet with such a procedure we would lose all information about the efficient intertemporal ecosystem allocation and about the efficient ecosystem prices. We therefore consider, instead, a social planner who maximizes the present value of the representative consumers utility subject to (1), (2) and (4) - (8).

\section{Proposition 3.}

Let $\{\tau(t)\}$ be a tax policy that results in a general competitive equilibrium of the IEES ${ }^{11}$ characterized by positive market clearing prices in the economy, $\left(p_{h}, p_{\ell}, p_{y}\right)$, and in the ecosystem, $\left(\pi_{r}, \pi_{z}\right)$, at each point in time.

(i) The equilibrium allocation is efficient if and only if $\{\tau(t)\}$ satisfies

$$
\tau(t)=\pi_{z}(t) \quad \text { for all } t \geq 0
$$

(ii) If labor and the resource are chosen as the numeraire in the economy and the ecosystem, respectively, there exists a function $K: \mathbb{R}_{+}^{2} \longrightarrow \mathbb{R}_{+}$, specified in the Appendix, such that the efficient harvesting tax rate is equal to

$$
\tau(t)=K\left[n_{1}(t), n_{2}(t)\right] \quad \text { for all } t \geq 0
$$

In Proposition 3 equation (22) represents the efficient taxing rule. To interprete that rule recall that 'harvesting' of prey biomass by ecosystem predators is costless whereas human predators incur positive marginal harvesting costs, $c_{h}$. The prey biomass itself is a free commodity in the economy if no tax is levied but in the ecosystem that biomass is scarce $\left(\pi_{z}>0\right)$. According to (22) efficiency requires to account for the ecosystem price of biomass, $\pi_{z}$, when biomass is to be efficiently priced in the economy $p_{h}=c_{h}+\tau=c_{h}+\pi_{z}$. The value of one unit of biomass in the ecosystem, $\pi_{z}$, is the opportunity cost of consuming that unit in the economy. With zero marginal harvesting costs in the economy, efficiency would require the prices of biomass in the economy and in the ecosystem to be the same.

Proposition 3(ii) states that at each point in time the efficient tax rate (= the ecosystem price of prey biomass) is determined by both species' populations at that point in informative analytical results.

\footnotetext{
${ }^{11}$ The existence of such an equilibrium is secured for a large range of tax policies.
} 
time. As long as these populations change on their path toward the efficient stationary state, the efficient tax rate will also change, in general. Equation (23) is also very useful from an analytical or technical point of view. Once $\tau$ is known to be a function of $n_{1}$ and $n_{2}$ we can insert $\tau$ from (22) in (11c) and insert the resulting term for $h$ in the population growth functions (21). These functions now determine the socially optimal time path for the populations which we denote by $n_{1}^{*}(t)$ and $n_{2}^{*}(t)$. With this information we immediately get $\tau^{*}(t)=K\left[n_{1}^{*}(t), n_{2}^{*}(t)\right]$, and the complete time path for $\left[h^{*}(t), y^{*}(t), \ell_{h}^{*}(t), \ell_{y}^{*}(t), r_{1}^{*}(t), z_{1}^{*}(t), z_{2}^{*}(t)\right]$ can be calculated by plugging $\tau^{*}(t)$ into $(11 \mathrm{c})$ (11f) and $h^{*}(t)$ into (20a)-(20c).

\section{Economic-ecological dynamics in numerical analysis}

Quite evidently, the complexity of the differential equations involved prevents us from characterizing the dynamics of the integrated economy-ecosystem model analytically. This is not only true for the dynamics pertaining to the efficient intertemporal tax policy but also for the dynamics of any other tax policy. We will therefore resort to numerical specifications of parameters to be used for describing and comparing different harvesting scenarios. The numerical examples are computed with the help of the software Mathematica. ${ }^{12}$ In all subsequent calculations the parameters are specified as follows: $c_{y}=1, c_{h}=0.007, \delta=0.05$, $\bar{r}=1, \bar{\ell}=1, \bar{z}_{1}=\bar{z}_{2}=1, \gamma_{1}=0.01, \gamma_{2}=1, \kappa=0.2, \varepsilon=1 / 10, \phi_{1}=\phi_{2}=\psi_{1}=0.5, \sigma_{1}=1$ and $\sigma_{2}=100$. These parameter values are not based on empirical data.

Before the results are presented and discussed in detail, a few remarks on the procedure and on some general characteristics of the numerical dynamics are in order. We will present the numerical dynamics of the following harvesting scenarios:

(i) In the zero-harvesting scenario no harvesting takes place at all, ${ }^{13}$ i.e. $h \equiv 0$. In that case the economy unilaterally depends on the ecosystem via $n_{2}$.

(ii) In the laissez-faire harvesting scenario biomass is harvested in the absence of any nature conservation policy, i.e. $\tau \equiv 0$.

(iii) In the efficient-harvesting scenario the tax rate is set equal to $\tau=\pi_{z}$ as required by Proposition 3(i).

We will begin with the zero-harvesting scenario where the initial populations are fixed at $n_{1}(0)=10$ and $n_{2}(0)=1$ in a somewhat arbitrary way. We calculate the time path of

\footnotetext{
${ }^{12}$ The program for simulations is available from the authors upon request.

${ }^{13}$ This scenario can also be interpreted, approximately at least, as a tax policy with an extremely high tax rate, since (11c) implies $h \rightarrow 0$ for $\tau \rightarrow \infty$.
} 
the IEES toward its stationary state populations $n_{1}(0)=5050$ and $n_{2}(0)=2500$ and then take those numbers as the initial populations for a regime switch to laissez-faire harvesting. Next, that new scenario is characterized from time zero to its stationary state populations $n_{1}(0)=3338$ and $n_{2}(0)=1081$. Finally we use those numbers as initial populations in the efficient-harvesting scenario whose adjustment patterns and stationary-state properties are then investigated. With minor exceptions all variables will turn out to converge to their stationary state values in a monotone way.

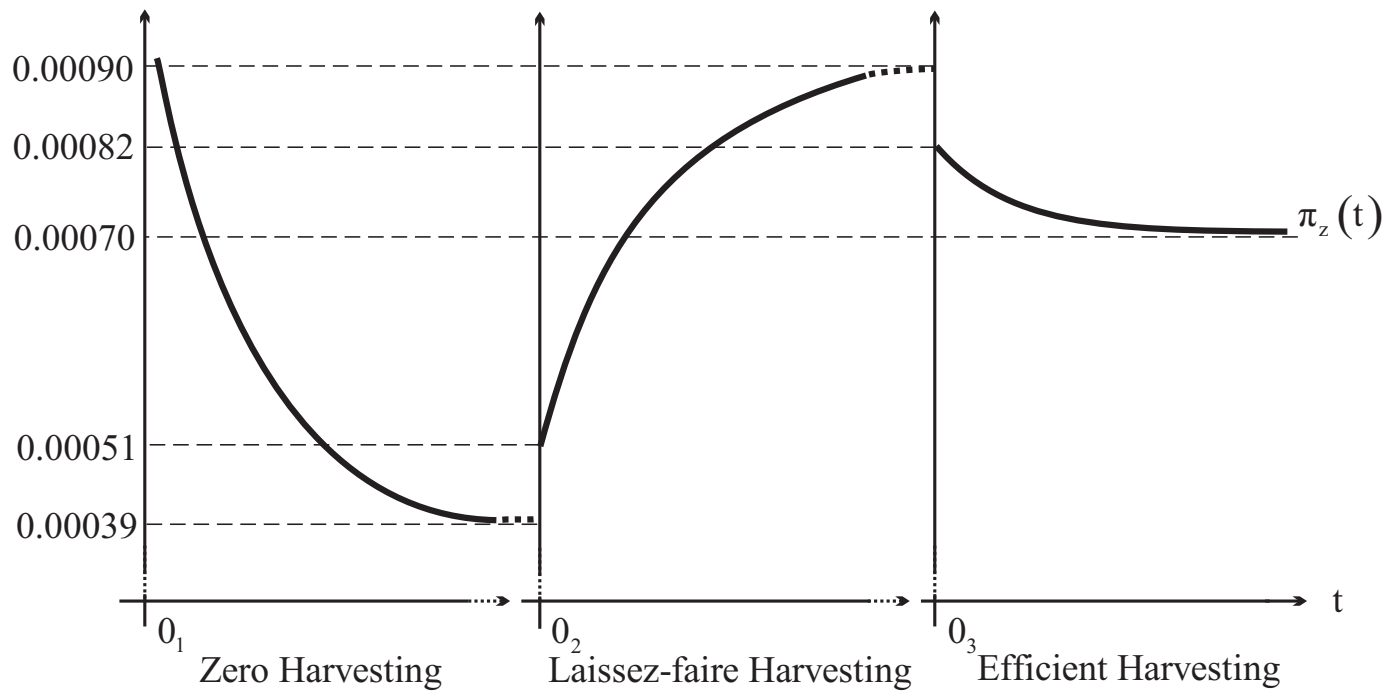

Figure 1a: Time paths of the ratio of the ecosystem price for prey biomass and the ecosystem price for land

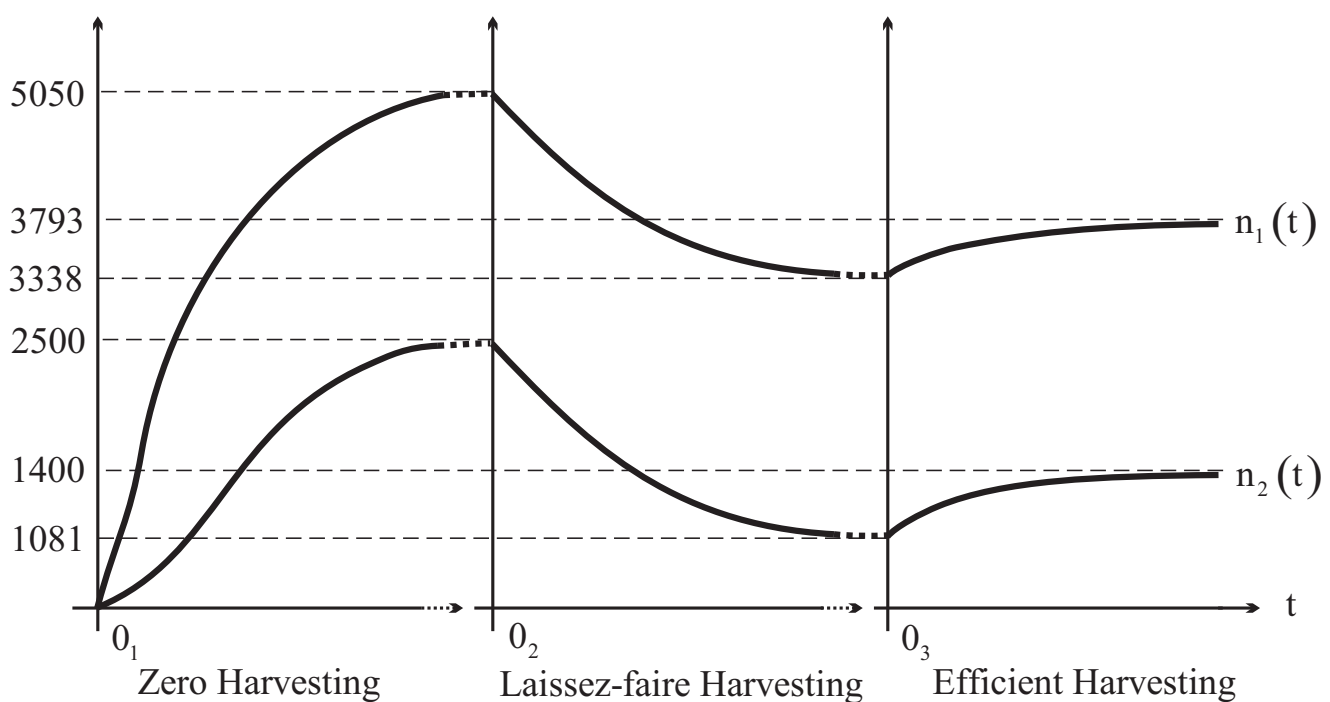

Figure $1 \mathrm{~b}$ : Time paths of populations

Figure 1: IEES dynamics in alternative harvesting scenarios

Figure 1 illustrates the three scenarios in a sequence and offers, at the same time, some principal results. ${ }^{14}$ Figures $1 \mathrm{a}$ and $1 \mathrm{~b}$ both consist of three panels with origins $0_{1}, 0_{2}$

\footnotetext{
${ }^{14}$ We have preferred to present freehand drawings in Figure 1 over graphs directly plotted from numerical
} 
and $0_{3}$, respectively, where in each quadrant the abscissa measures time from zero toward infinity. The left panel presents the zero-harvesting scenario, the middle one the laissezfaire harvesting scenario and the right panel the efficient-harvesting scenario. To provide further information on the comparison of scenarios, Table 4 supplements Figure 1 by displaying stationary state values as well as directions of change of the economic and ecological variables in all scenarios. We will now discuss the scenarios in more detail, one at a time.

\begin{tabular}{|c|c|c|c|c|c|c|}
\hline \multirow{6}{*}{ Variable } & \multicolumn{6}{|c|}{ Harvesting Scenario: } \\
\hline & \multicolumn{2}{|c|}{ Zero $(h=0)$} & \multicolumn{2}{|c|}{ Laissez-faire $(\tau=0)$} & \multicolumn{2}{|c|}{ Efficiency $\left(\tau=\pi_{z}\right)$} \\
\hline & Direction & Steady & Direction & Steady & Direction & Steady \\
\hline & & State & & State & & State \\
\hline & Change*) & Value & Change*) & Value & Change*) & Value \\
\hline & (1) & $(2)$ & (3) & $(4)$ & $(5)$ & $(6)$ \\
\hline \multicolumn{7}{|c|}{ ECOSYSTEM } \\
\hline$n_{1}$ & $\uparrow$ & 5050 & $\downarrow$ & 3338 & $\uparrow$ & 3793 \\
\hline$n_{2}$ & $\uparrow$ & 2500 & $\downarrow$ & 1081 & $\uparrow$ & 1400 \\
\hline$r_{1}$ & $\downarrow$ & 0.000198 & $\uparrow$ & 0.000299 & $\downarrow$ & 0.000264 \\
\hline$z_{2}$ & $\downarrow$ & 1 & $\downarrow \uparrow$ & 1 & $\uparrow \downarrow$ & 1 \\
\hline$z_{1}$ & $\uparrow$ & 0.495 & $\uparrow \downarrow \uparrow$ & 0.666 & $\downarrow \uparrow \downarrow$ & 0.621 \\
\hline$\omega_{1}$ & $\downarrow$ & $3.9 \cdot 10^{-6}$ & $\uparrow$ & $8.94 \cdot 10^{-6}$ & $\downarrow$ & $6.95 \cdot 10^{-6}$ \\
\hline$\omega_{2}$ & $\downarrow$ & 0.000390 & $\uparrow$ & 0.000894 & $\downarrow$ & 0.000695 \\
\hline$\pi_{z}$ & $\downarrow$ & 0.000392 & $\uparrow$ & 0.000897 & $\downarrow$ & 0.000695 \\
\hline \multicolumn{7}{|c|}{ ECONOMY } \\
\hline$h$ & $\rightarrow$ & 0 & $\rightarrow$ & 1143 & $\downarrow \uparrow$ & 953 \\
\hline$\ell_{h}$ & $\rightarrow$ & 0 & $\rightarrow$ & 0.8 & $\downarrow \uparrow$ & 0.667 \\
\hline$\ell_{y}$ & $\rightarrow$ & 1 & $\rightarrow$ & 0.2 & $\uparrow \downarrow$ & 0.333 \\
\hline$y$ & $\rightarrow$ & 1 & $\rightarrow$ & 0.2 & $\uparrow \downarrow$ & 0.333 \\
\hline$u$ & $\uparrow$ & 250 & $\uparrow \downarrow$ & 311 & $\downarrow \uparrow$ & 336 \\
\hline$p_{y}$ & $\rightarrow$ & 1 & $\rightarrow$ & 1 & $\rightarrow$ & 1 \\
\hline$p_{h}$ & $\rightarrow$ & - & $\rightarrow$ & 0.007 & $\downarrow \uparrow$ & 0.00769 \\
\hline
\end{tabular}

Table 4: Characterization of alternative harvesting scenarios in the IEES $\left.\left(\pi_{r}=p_{\ell} \equiv 1\right){ }^{*}\right)$ after the regime switch occured]

calculations with Mathemetica. The precise plots are disconnected, exhibit different scales and use up much space whereas freehand drawings compress the relevant qualitative information and thus facilitate the comparison of scenarios. The precise plots can be obtained from the authors upon request 


\subsection{Zero harvesting}

The left panel of Figure 1b depicts the development of the populations of both species over time. Starting from a relatively small initial population both species exhibit logistic growth. Interestingly, the prey species keeps growing from $n_{1}(0)=10$ toward its stationary state $n_{1}=5050$ despite continuous predation. The predator species grows faster than the prey species, since the ratio of populations increases from $\left[n_{2}(0) / n_{1}(0)\right]=0.1$ to $\left[n_{2}(\infty) / n_{1}(\infty)\right]=0.49$. The left panel of Figure 1a shows the development of relative scarcities in the ecosystem along the transition path to the stationary state: At the beginning, prey biomass is very scarce relative to land (or conversely, land is very abundant relative to prey biomass) but it then becomes more and more abundant as the ecosystem moves toward its stationary state. Additional qualitative information about the directions of change of the relevant variables is provided in column (1) of Table 4.

To explore the driving force of this scenario observe first that with increasing populations the representative organisms' resource endowments ${ }^{15}$ decrease $\left(\omega_{1} \downarrow\right.$ and $\left.\omega_{2} \downarrow\right)$. The predator reduces its demand for prey biomass $\left(z_{2} \downarrow\right)$ since although the price of prey biomass declines the predator's budget shrinks even faster.

The prey partly compensates its loss of resource endowment through supplying more own biomass $\left(\begin{array}{ll}z_{1} & \uparrow\end{array}\right)$ but it cannot avoid cutting back its uptake of land services $\left(r_{1} \downarrow\right)$. Hence $\mathrm{d} b_{1}=\frac{\partial b_{1}}{\partial r_{1}} \mathrm{~d} r_{1}+\frac{\partial b_{1}}{\partial z_{1}} \mathrm{~d} z_{1}<0$ because $\mathrm{d} r_{1}<0$ and $\mathrm{d} z_{1}>0$. The immediate consequence of a rise in the supply of prey biomass $\left(z_{1} \uparrow\right)$ and a decreasing demand for prey biomass $\left(z_{2} \downarrow\right)$ is a drop in price $\left(\pi_{z} \downarrow\right)$ as illustrated in Figure 1a, left panel.

After the stationary state of the zero-harvesting scenario has been reached we switch to laissez-faire harvesting. That is, we now take the stationary-state populations $n_{1}=5050$ and $n_{2}=2500$ of the zero-harvesting scenario as the initial populations of the laissez-faire harvesting scenario characterized by $\tau \equiv 0$.

\subsection{Laissez-faire harvesting}

With our numerical specification of parameters introduced above, (11c) is turned into $h=$ 1143. The consequence is a continuous decline in both populations as shown in the middle panel of Figure 1b. With increasing $t$ the negative growth of populations levels off and the populations converge to their stationary-state values $n_{1}=3338$ and $n_{2}=1081$, respectively. The relative decline of the predator population $(57 \%)$ is stronger than that of the prey population $(34 \%)$. As in the zero-harvesting scenario, the change in the relative price of

\footnotetext{
${ }^{15}$ Since $\pi_{r} \equiv 1$, the endowment $\omega_{i}$ is equal to 'capital income' $\pi_{r} \omega_{i}$.
} 
prey biomass, $\pi_{z}$, is inverse to the development of populations. The middle panel of Figure 1a shows a significant increase over time in the relative price of prey biomass caused by human harvest.

To explore the changes induced by the regime switch it is necessary to distinguish changes at the time of switching and changes occurring after the switch. The changes at the point in time of the regime switch are the changes between the allocation of the IEES in a stationary state of the zero-harvesting scenario and the allocation at the point in time $(t=0)$ when laissez-faire harvesting is introduced. The changes after the switching time are the changes of all variables on their time path from $t=0+\epsilon, \epsilon$ arbitrarily small, toward $t=\infty$.

Consider first the instantaneous allocative displacement effects at the time of regime switch. Turning from $h=0$ to $h>0$ constitutes an exogenous shock to both the economy and the ecosystem. The allocative impact is listed in column (4) of Table 4 . In the ecosystem $n_{1}, n_{2}, r_{1}, \omega_{1}$ and $\omega_{2}$ remain unchanged but the stationary state price $\pi_{z}$ jumps up from 0.00039 to 0.00051 (as shown in Figure 1a). As a consequence, $z_{1}$ rises from 0.495 to 0.609 to accommodate for harvesting but the hike in $\pi_{z}$ forces organism 2 to reduce its demand for prey biomass from $z_{2}=1$ to $z_{2}=0.774$. Thus both species suffer from harvesting: the prey now has to sacrifice own biomass to the humans ${ }^{16}$ while the rise in $\pi_{z}$ reduces the real income of all ecosystem predators and hence their prey biomass intake.

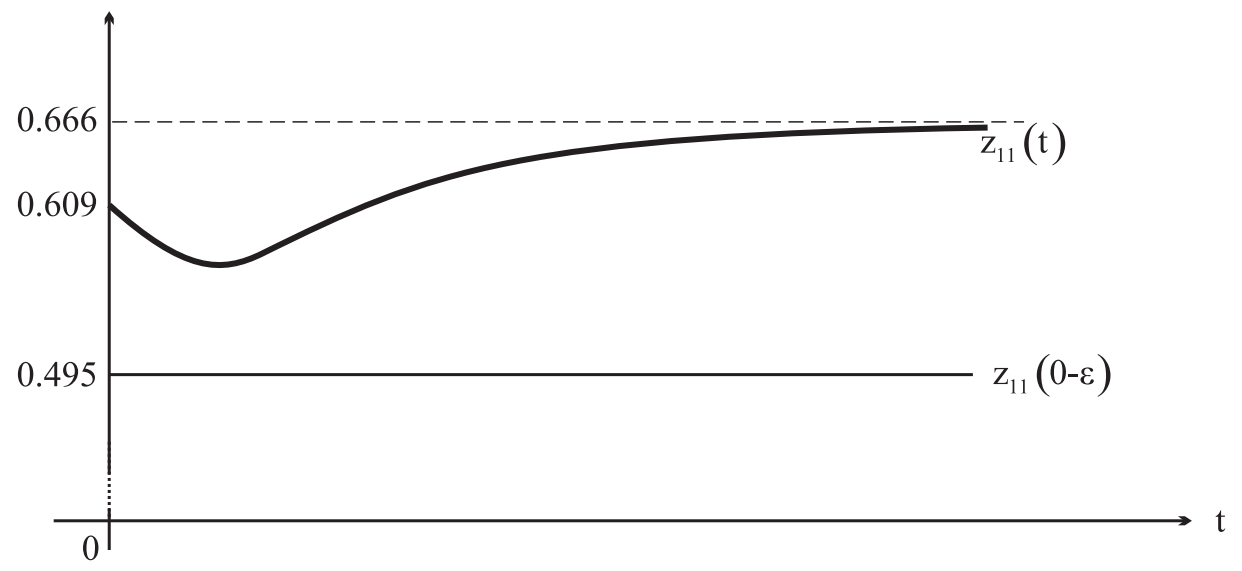

Figure 2: The time path of biomass supply in the laissez-faire harvesting scenario

Consider now the time interval immediately following $t=0$. Due to reduced consump-

\footnotetext{
${ }^{16}$ When the prey sacrifices own biomass to the predator it receives 'biomass income' in exchange which it can spend on land services, $r_{1}$. In contrast, humans do not pay the prey for the biomass they harvest. Yet it may be considered a consolation for the prey that the increase in $\pi_{z}$ raises its biomass income, ceteris paribus.
} 
tion of prey biomass the predator population shrinks, and the prey population shrinks, too, due to harvesting. The shrinking populations trigger two partial effects:

(i) The endowment effect: All organisms' resource endowments grow which induces, ceteris paribus, an increase in $r_{1}, z_{2}, \pi_{z}$ and a reduction in $z_{1}$ via (18a) - (18c) and (19).

(ii) The harvesting effect: Although $h$ is time invariant, the per-capita harvest $\tilde{h}:=h / n_{1}$ grows when the population $n_{1}$ shrinks. Ceteris paribus, this decline in $\tilde{h}$ reduces $r_{1}$, leaves $z_{2}$ unchanged and increases $\pi_{z}$ via $(18 \mathrm{a})$ - (18c) and (19).

With respect to $r_{1}$ and $z_{1}\left[z_{2}\right.$ and $\left.\pi_{z}\right]$ both effects [do not] point into opposite directions. Hence the variables $z_{2}$ and $\pi_{z}$ are increasing on their paths to the stationary state. In case of $r_{1}$ the endowment effect dominates the harvesting effect such that $r_{1}$ is also strictly increasing. ${ }^{17}$ This feature of dominance emerges for $z_{1}$ only after some time. As shown in Figure 2, $z_{1}$ first takes a dip during some time interval immediately following $t=0$ implying that during that interval the endowment effect is stronger than the harvesting effect. Note, however, that in the laissez-faire harvesting regime $z_{1}$ is always greater than in the preceding zero-harvesting scenario.

The development of the economy during its transition to the stationary state is easily described. Except for utility $u$, the economic variables are as listed in column (4) of Table 4 along the entire time path. ${ }^{18}$ The utility strictly decreases from $u=408$ at $t=0$ to its stationary-state value $u=311$.

\subsection{Efficient harvesting}

The third and last numerical exercise takes as its starting point the stationary-state populations $n_{1}=3338$ and $n_{2}=1081$ of the laissez-faire harvesting scenario and then switches to the efficient-harvesting regime. Consider first the instantaneous effects at the time of regime switch: As in the preceding section, the variables $n_{1}, n_{2}, r_{1}, \omega_{1}$ and $\omega_{2}$ remain unchanged but $z_{2}$ rises from 1 to $1.098, z_{1}$ drops from 0.666 to 0.633 and $\pi_{z}$ from 0.000897 to 0.000816. The reason for these jumps is that it is efficient to drastically reduce harvesting from $h=1143$ to $h=926$ at $t=0$. Clearly, less harvesting goes hand in hand with less labor used for harvesting $\ell_{h}(0)=0.648$ and more labor $\ell_{y}(0)=0.35$ is employed to increase

\footnotetext{
${ }^{17}$ This development of $r_{1}$ is forced by the equilibrium condition (20a), of course. As long as $n_{1}$ shrinks, $r_{1}$ is bound to increase.

${ }^{18}$ As observed in Section 3.1, in less simple models with ecosystem services not being additively separable components of the consumers' utility all these variables would respond, in general, to changes in the provision of these services.
} 
the production of the consumer good $y(0)=0.35$. These switches turn out to cause a loss in utility since $u=300$ at $t=0$.

Figure 1 shows that after the regime switch the growth of the populations $n_{1}$ and $n_{2}$ and the development of the relative price $\pi_{z}$ are as in the zero-harvesting scenario, qualitatively speaking: both populations grow and the relative price $\pi_{z}$ diminishes. Interestingly, it is efficient to keep the harvest small at the beginning and to increase it successively toward its stationary-state value $h=953$. Per-capita harvesting decreases from $\tilde{h}=0.2776$ at $t=0$ to its stationary-state level $\tilde{h}=0.2513$. This development is controlled by taxation. As in case of the laissez-faire scenario the endowment and the harvesting effect drive the changes of $r_{1}, z_{1}$ and $z_{2}$. But in contrast to the last subsection they are opposite in sign since the populations increase (causing a reduction in endowments) and the per-capita harvesting rate decreases. Consequently, after the displacement effects at the time of regime switch all transactions $\left(r_{1}, z_{1}, z_{2}\right)$ in the ecosystem and the ecosystem price $\pi_{z}$ are strictly decreasing.

Since $\tau=\pi_{z}$ and since $\pi_{z}$ decreases in the efficient-harvesting scenario (as shown in Figure 1a) biomass becomes less expensive in the economy and therefore the amount of biomass harvested increases. Nonetheless, it stays well below the laissez-faire level of harvest, $n_{1}=1143$, at all times. This is the reason, of course, for the population growth and for the fact that the stationary-state populations are now greater than in the laissezfaire regime. As expected, the efficient stationary-state populations are smaller than in the absence of harvesting, since it is efficient to harvest positive amounts of biomass.

\section{Concluding remarks}

The main methodological contribution of the present paper is to place harvesting in the context of a microfounded IEES where both submodels, the ecosystem and the economy, are treated at the same level of structural complexity. Each submodel is inhabited by optimizing individual agents and possesses its own allocation mechanism as a means to 'coordinate' decentralized transactions. In this way, shocks in the economy with an impact on harvesting trigger price changes and adjustments of transactions within the ecosystem which eventually translate in feedback effects on the economy.

Our numerical analysis of the harvesting scenarios in Section 4 follows exactly the methodological and programmatic approach of David Pearce (1976, p. 320) taken as our introductory motto, namely that "... it is a matter of weighing costs and benefits of taking action, whether the action is the "inert" one of leaving resources alone in order to conserve 
them, or whether it involves exploiting a resource ... for so-called material ends". ${ }^{19}$ As compared to conventional macro-level bioeconomic models of harvesting, the present approach explicitly models intra-ecosystem activities and actually uses them to explain the interaction between harvesting and population dynamics. It allows to assess in more detail ecosystem scarcities and values, how they are affected by human encroachments and how these values have to be taken into account to optimize the resource use. In that way, our approach promises to guide more precisely, and hence improve, the necessary process of weighing the costs and benefits of harvesting and the human use of natural resources, more generally.

For convenience of exposition and analytical tractability, we have kept both submodels very simple. Yet it is clear that nowadays computing capacity does not seriously constrain large-scale numerical calculations as the blooming industry of the computable general equilibrium analysis of economic models demonstrates. We believe that based on the methodology of the present paper it will also be possible in the future to develop more realistic large-scale computable general equilibrium models of the ecosystem. Finnoff and Tschirhart (2003a, 2003b) already showed in different but related frameworks that the callibration of fairly complex general equilibrium models of the ecosystem is both possible and fruitful. The ultimate goal is, of course, to construct a computable general equilibrium model of both the ecosystem and the economy which are then combined to a hybrid computable general equilibrium of the IEES. Idealy, such a model would be capable to compute efficient ecosystem prices and efficient harvesting tax rates or harvesting quotas. One would then also want to consider including into that hybrid model ecological-economic interdependencies caused by pollution and by land conversion.

\section{References}

Christiaans T., Eichner T. and R. Pethig (2006), A micro-level 'consumer approach' to species population dynamics, Natural Resource Modeling, in press.

Clark C.W. (1997), Mathematical bioeconomics: The optimal management of renewable resources, Wiley \& Sons, New York et al.

Crocker T. D. and J. Tschirhart (1992), Ecosystems, externalities and economies, Environmental and Resource Economics 2, 551-567.

\footnotetext{
${ }^{19}$ Making progress in weighing the costs and benefits of taking action was so important to Pearce that he did not even shy away from "... an exercise in "applied heroics" in the level of generality it seeks" (Pearce 1976, p. 323). In that perspective, our approach may be considered an exercise in "analytical heroics".
} 
Deaton A. (1981), Optimal taxes and the structure of preferences, Econometrica 49, 12451260.

Eggert H. (1998), Bioeconomic analysis: The case of fisheries, Environmental and Resource Economics 11, 399-411.

Eichner T. and R. Pethig (2003), The impact of scarcity and abundance in food chains on species population dynamics, Natural Resource Modeling 16, 259-303.

Eichner T. and R. Pethig (2006a), An analytical foundation of the ratio-dependent predatorprey model, Journal of Bioeconomics, in press.

Eichner T. and R. Pethig (2006b), A microfoundation of predator-prey dynamics, Natural Resource Modeling, in press.

Eichner T. and R. Pethig (2006c), Pricing the ecosystem and taxing ecosystem services: a general equilibrium approach, Discussion paper, University of Siegen.

Finnoff D. and J. Tschirhart (2003a), Harvesting in an eight-species ecosystem, Journal of Environmental Economics and Management 45, 589-611.

Finnoff D. and J. Tschirhart (2003b), Protecting an endangered species while harvesting its prey in a general equilibrium ecosystem model, Land Economics 79, 160-180.

Hannon B. (1976), Marginal product pricing in the ecosystem, Journal of Theoretical Biology 56, 253-267.

Hoekstra J. and J.C.J.M. van den Bergh (2005), Harvesting and conservation in a predatorprey system, Journal of Economic Dynamics and Control 29, 1097-1120.

Lima S.L. and L.M. Dik (1990), Behavioral decisions made under the risk of predation: A review and prospectus, Canadian Journal of Zoology 68, 619-640.

Pearce D. (1976), Environmental protection, recycling, and the international materials economy, in: I. Walter (ed.), Studies in International Environmental Economics, Wiley \& Sons, New York et al., 319-347.

Stephens D.W. and J.R. Krebs (1986), Foraging Theory, Princeton University Press, Princeton.

Ströbele W.J. and H. Wacker (1995), The economics of harvesting predator-prey systems, Journal of Economics 61, 65-81.

Tschirhart J. (2000), General equilibrium of an ecosystem, Journal of Theoretical Biology 203, 13-32. 


\section{Appendix}

\section{Proof of Proposition 1:}

The partial derivative of (21a) with respect to $n_{1}$ evaluated at $\dot{n}_{1}=0$ is

$$
\left.\frac{\partial \dot{n}_{1}}{\partial n_{1}}\right|_{\dot{n}_{1}=0}=-\left(\frac{\bar{r}}{n_{1}}\right)^{\phi_{1}} \Upsilon^{\psi_{1}}\left(\bar{z}_{1}-\frac{h}{n_{1}}\right) \frac{1}{n_{1}\left(n_{1} \sigma_{1}+n_{2} \sigma_{2} \psi_{1}\right)} \cdot \tilde{M}\left(h, n_{1}, n_{2}\right)
$$

where

$$
\begin{aligned}
\tilde{M}\left(h, n_{1}, n_{2}\right) & :=\phi_{1}\left(\bar{z}_{1}-\frac{h}{n_{1}}\right)\left(n_{1} \sigma_{1}+n_{2} \sigma_{2} \psi_{1}\right)-h n_{1} \sigma_{2} \psi_{1} \phi_{1}-\frac{n_{1} n_{2} \sigma_{2}^{2} \psi_{1} \phi_{1}\left(\phi_{1}+\psi_{1}\right)}{n_{1} \sigma_{1} \psi_{1}+n_{2} \sigma_{2}\left(\phi_{1}+\psi_{1}\right)}, \\
\Upsilon & :=\frac{\left(n_{1} \sigma_{1}+n_{2} \sigma_{2}\right) \psi_{1}\left(\bar{z}_{1}-\frac{h}{n_{1}}\right)}{n_{1} \sigma_{1} \psi_{1}+n_{2} \sigma_{2}\left(\phi_{1}+\psi_{1}\right)} .
\end{aligned}
$$

Under the assumption $M(\tau):=\tilde{M}\left[H(\tau), F^{1}(\tau), F^{2}(\tau)\right]>0$ it is true that $\left.\frac{\partial \dot{n}_{1}}{\partial n_{1}}\right|_{\dot{n}_{1}=0}<0$. Differentiation of (21a) with respect to $n_{2}$ yields

$$
\left.\frac{\partial \dot{n}_{1}}{\partial n_{2}}\right|_{\dot{n}_{1}=0}=-\left(\frac{\bar{r}}{n_{1}}\right)^{\phi_{1}} \psi_{1} \Upsilon^{\psi_{1}-1} \cdot \frac{n_{1} \sigma_{1}^{2} \psi_{1} \phi_{1}\left(\bar{z}_{1}-\frac{h}{n_{1}}\right)}{\left[n_{1} \sigma_{1} \psi_{1}+n_{2} \sigma_{2}\left(\phi_{1}+\psi_{1}\right)\right]^{2}}<0 .
$$

The partial derivatives of $(21 \mathrm{~b})$ evaluated at $\dot{n}_{2}=0$ are

$$
\begin{aligned}
&\left.\frac{\partial \dot{n}_{2}}{\partial n_{1}}\right|_{\dot{n}_{2}=0}= \phi_{2} \Omega^{\phi_{2}-1} \cdot\left\{\frac{h \phi_{1} \sigma_{2}}{n_{1}^{2}\left[\sigma_{1} \psi_{1}+\frac{n_{2}}{n_{1}} \sigma_{2}\left(\phi_{1}+\psi_{1}\right)\right]}+\frac{\phi_{1} \sigma_{2}\left(\bar{z}_{1}-\frac{h}{n_{1}}\right)}{\left[\sigma_{1} \psi_{1}+\frac{n_{2}}{n_{1}} \sigma_{2}\left(\phi_{1}+\psi_{1}\right)\right]^{2}}\right. \\
&\left.\cdot \frac{n_{2} \sigma_{2}\left(\phi_{1}+\psi_{1}\right)}{n_{1}^{2}}\right\}>0, \\
&\left.\frac{\partial \dot{n}_{2}}{\partial n_{2}}\right|_{\dot{n}_{2}=0}=-\phi_{2} \Omega^{\phi_{2}-1} \sigma_{2}\left(\phi_{1}+\psi_{1}\right) \cdot \frac{n_{1} \phi_{1} \sigma_{2}\left(\bar{z}_{1}-\frac{h}{n_{1}}\right)}{\left[n_{1} \sigma_{1} \psi_{1}+n_{2} \sigma_{2}\left(\phi_{1}+\psi_{1}\right)\right]^{2}}<0,
\end{aligned}
$$

where $\Omega:=\frac{n_{1} \phi_{1} \sigma_{2} \bar{z}_{1}-\frac{h}{n_{1}}}{n_{1} \sigma_{1} \psi_{1}+n_{2} \sigma_{2}\left(\phi_{1}+\psi_{1}\right)}$. Hence, the Jacobian of the differential equations (21) evaluated at $\left[n_{1}=F^{1}(\tau), n_{2}=F^{2}(\tau)\right]$ have the following pattern of signs:

$$
\operatorname{sgn}(J)=\operatorname{sgn}\left(\begin{array}{ll}
\partial \dot{n}_{1} / \partial n_{1} & \partial \dot{n}_{1} / \partial n_{2} \\
\partial \dot{n}_{2} / \partial n_{1} & \partial \dot{n}_{2} / \partial n_{2}
\end{array}\right)=\left(\begin{array}{ll}
- & - \\
+ & -
\end{array}\right) .
$$

Therefore, $\operatorname{Tr}(J)<0$ and $|J|>0$, implying that the equilibrium $\left[n_{1}=F^{1}(\tau)>0, n_{2}=F^{2}(\tau)\right]$ is locally asymptotically stable by the Routh-Hurwitz criterion. 


\section{Proof of Proposition 2:}

Since the results of Table 2 follow directly from differentiation of (11) with respect to $\tau$, it remains to prove the results of Table 3 . We begin with the comparative dynamics of $n_{1}$ and $n_{2}$. First we set $\dot{n}_{2}=0$ and solve $(21 \mathrm{~b})$ with respect to $n_{2}$ to get

$$
n_{2}=n_{1} \cdot\left[\frac{\phi_{1}\left(\bar{z}_{1}-\tilde{h}\right)}{\left(\phi_{1}+\psi_{1}\right) \gamma_{2}^{\frac{1}{\phi_{2}}}}-\frac{\sigma_{1} \psi_{1}}{\sigma_{2}\left(\phi_{1}+\psi_{1}\right)}\right] \text {. }
$$

Next, we set $\dot{n}_{1}=0$ and use (25) to obtain

$$
n_{1}=\frac{\bar{r}}{\gamma_{1}} \cdot\left[\frac{\psi_{1}}{\phi_{1}+\psi_{1}} \cdot\left(\frac{\sigma_{1} \gamma_{2}^{\frac{1}{\phi_{2}}}}{\sigma_{2}+\bar{z}_{1}-\tilde{h}}\right)\right]^{\frac{\psi_{1}}{\phi_{1}}}
$$

Defining

$$
G(\tilde{h})=\frac{\bar{r}}{\gamma_{1}} \cdot\left[\frac{\psi_{1}}{\phi_{1}+\psi_{1}} \cdot\left(\frac{\sigma_{1} \gamma_{2}^{\frac{1}{\phi_{2}}}}{\sigma_{2}+\bar{z}_{1}-\tilde{h}}\right)\right]^{\frac{\psi_{1}}{\phi_{1}}}
$$

the stationary-state population level $n_{1}$ is implicitly determined by $n_{1}=G(\tilde{h})$. The function $G$ is strictly decreasing, $G_{\tilde{h}}<0$, and the elasticity of stationary-state population with respect to harvesting $\varepsilon\left(n_{1}, \tilde{h}\right):=G_{\tilde{h}} \cdot(\tilde{h} / G)$ satisfies

$$
\varepsilon\left(n_{1}, \tilde{h}\right)>-1
$$

Making use of the definition $\tilde{h}=h / n_{1}$ we clearly define $F^{1}$ by the condition

$$
n_{1}=F^{1}(\tau) \quad \Longleftrightarrow \quad n_{1}=G\left(\frac{H(\tau)}{n_{1}}\right)
$$

where $H(\tau):=\frac{\bar{\ell}(1-\kappa)}{c_{h}+\kappa \tau c_{y}}($ compare $(11 \mathrm{c}))$. To establish the derivative of $F^{1}$ we calculate the derivative of the last equation as

$$
\mathrm{d} n_{1}=-G_{\tilde{h}} \frac{h}{n_{1}^{2}} \mathrm{~d} n_{1}+G_{\tilde{h}} \frac{H_{\tau}}{n_{1}} \mathrm{~d} \tau
$$

and hence

$$
F_{\tau}^{1}=\frac{\mathrm{d} n_{1}}{\mathrm{~d} \tau}=\frac{G_{\tilde{h}} H_{\tau}}{n_{1}\left[1+\varepsilon\left(n_{1}, \tilde{h}\right)\right]},
$$

which establishes $F_{\tau}^{1}>0$ due to $G_{\tilde{h}}<0,(28)$ and $H_{\tau}<0$. Consider now the stationarystate population $n_{2}$. In view of $(25)$ and $n_{1}=F^{1}(\tau)$ we obtain

$$
n_{2}=F^{2}(\tau)=F^{1}(\tau) \cdot\left[\frac{\phi_{1} \bar{z}_{1}}{\left(\phi_{1}+\psi_{1}\right) \gamma_{2}^{\frac{1}{\phi_{2}}}}-\frac{\sigma_{1} \psi_{1}}{\sigma_{2}\left(\phi_{1}+\psi_{1}\right)}\right]-\frac{\phi_{1}}{\left(\phi_{1}+\psi_{1}\right) \gamma_{2}^{\frac{1}{\phi_{2}}}} \cdot H(\tau) .
$$


Since the bracketed term $[\cdot]$ is positive, we readily infer $F_{\tau}^{2}>0$. Thus, we have shown:

$$
\frac{\mathrm{d} n_{1}}{\mathrm{~d} \tau}=F_{\tau}^{1}>0, \quad \frac{\mathrm{d} n_{2}}{\mathrm{~d} \tau}=F_{\tau}^{2}>0 .
$$

Next, we differentiate (13) and (20a) to obtain

$$
\begin{aligned}
\frac{\mathrm{d} \omega_{1}}{\mathrm{~d} \tau} & =-\frac{\sigma_{1}^{2} \bar{r}}{\left(\sigma_{1} n_{1}+\sigma_{2} n_{2}\right)^{2}} \cdot \frac{\mathrm{d} n_{1}}{\mathrm{~d} \tau}<0, \quad \frac{\mathrm{d} \omega_{2}}{\mathrm{~d} \tau}=-\frac{\sigma_{1} \sigma_{2} \bar{r}}{\left(\sigma_{1} n_{1}+\sigma_{2} n_{2}\right)^{2}} \cdot \frac{\mathrm{d} n_{2}}{\mathrm{~d} \tau}<0, \\
\frac{\mathrm{d} r_{1}}{\mathrm{~d} \tau} & =-\frac{\bar{r}}{\left(n_{1}\right)^{2}} \cdot \frac{\mathrm{d} n_{1}}{\mathrm{~d} \tau}<0 .
\end{aligned}
$$

Substituting $n_{2}$ from (25) in (20c) establishes

$$
z_{2}=\left(\gamma_{2}\right)^{\frac{1}{\phi_{2}}} \quad \text { and hence } \quad \frac{\mathrm{d} z_{2}}{\mathrm{~d} \tau}=0 .
$$

Since $\pi_{r}=1$ we obviously have $\mathrm{d} \pi_{r} / \mathrm{d} \tau=0$ and differentiation of (18c) yields

$$
\frac{\mathrm{d} \pi_{z}}{\mathrm{~d} \tau}=\frac{\pi_{r}}{z_{2}} \cdot \frac{\mathrm{d} \omega_{2}}{\mathrm{~d} \tau}<0
$$

To determine the comparative dynamic effect with respect to $z_{1}$ we combine (8) and (25) to get

$$
z_{1}=\tilde{h}+\left[\frac{\phi_{1}\left(\bar{z}_{1}-\tilde{h}\right)}{\left(\phi_{1}+\psi_{1}\right) \gamma_{2}^{\frac{1}{\phi_{2}}}}-\frac{\sigma_{1} \psi_{1}}{\sigma_{2}\left(\phi_{1}+\psi_{1}\right)}\right] \cdot z_{2} .
$$

Inserting $z_{2}$ from (32) and differentiating of (34) yields

$$
\frac{\mathrm{d} z_{1}}{\mathrm{~d} \tau}=\frac{\psi_{1}}{\phi_{1}+\psi_{1}} \cdot \frac{\mathrm{d} \tilde{h}}{\mathrm{~d} \tau}<0 \quad \text { where } \quad \frac{\mathrm{d} \tilde{h}}{\mathrm{~d} \tau}=\frac{\mathrm{d} h}{n_{1} \mathrm{~d} \tau}-\frac{h \mathrm{~d} n_{1}}{n_{1}^{2} \mathrm{~d} \tau}<0
$$

\section{Proof of Proposition 3:}

(i) The efficient allocation of our economy-ecosystem model (1) - (8) is obtained by solving the Lagrangian

$$
\begin{aligned}
\mathcal{L} & =y^{\kappa} h^{1-\kappa}+\varepsilon n_{2}+\lambda_{y}\left(\frac{\ell_{y}}{c_{y}}-y\right)+\lambda_{h}\left(\frac{\ell_{h}}{c_{h}}-h\right) \\
& +\lambda_{\ell}\left(\bar{\ell}-\ell_{y}-\ell_{h}\right)+\lambda_{z}\left(n_{1} z_{1}-h-n_{2} z_{2}\right)+\lambda_{r}\left(\bar{r}-n_{1} r_{1}\right) \\
& +\beta_{1} n_{1}\left[r_{1}^{\phi_{1}}\left(\bar{z}_{1}-z_{1}\right)^{\psi_{1}}-\gamma_{1}\right]+\beta_{2} n_{2}\left(z_{2}^{\phi_{2}}-\gamma_{2}\right) .
\end{aligned}
$$

We apply the standard procedure of deriving first order conditions from (36) and match them with the first order conditions (10) and (17) pertaining to the optimization 
programs of the economic agents and organisms, respectively. As a result we specify the vector of prices $\left(p_{h}, p_{\ell}, p_{y}, \pi_{r}, \pi_{z}\right)$ and the tax rate $\tau$ as

$$
p_{\ell}=\lambda_{\ell}=\pi_{r}=\lambda_{r} \equiv 1, \quad p_{y}=\lambda_{y}=c_{y}, \quad p_{h}-\tau=\lambda_{h}=c_{h}, \quad \tau=\pi_{z}=\lambda_{z},
$$

where $\lambda_{y}, \lambda_{\ell}, \lambda_{r}, \lambda_{y}$ and $\lambda_{z}$ are the values attained by the Lagrange multipliers in the solution of (36).

(ii) Accounting for the equality $\tau=\pi_{z}$ we insert $h=H(\tau)$ into (19) and obtain

$$
\tau^{2}+\frac{c_{y} n_{1} \phi_{1} \bar{z}_{1}-\zeta \kappa c_{y}}{c_{h} n_{1} \phi_{1} \bar{z}_{1}} \cdot \tau-\frac{\phi_{1} \bar{\ell}(1-\kappa)+\zeta c_{h}}{c_{h} n_{1} \phi_{1} \bar{z}_{1}}=0
$$

where $\zeta:=n_{1} \psi_{1}\left(\frac{\sigma_{1} \bar{r}}{\sigma_{1} n_{1}+\sigma_{2} n_{2}}\right)+n_{2}\left(\phi_{1}+\psi_{1}\right)\left(\frac{\sigma_{2} \bar{r}}{\sigma_{1} n_{1}+\sigma_{2} n_{2}}\right)$. (38) is a quadratic function in $\tau$ whose solution is ${ }^{20}$

$$
\begin{aligned}
\tau & =\frac{\zeta \kappa c_{y}-c_{h} n_{1} \phi_{1} \bar{z}_{1}}{2 c_{h} n_{1} \phi_{1} \bar{z}_{1}}+\sqrt{\left(\frac{\zeta \kappa c_{y}-c_{h} n_{1} \phi_{1} \bar{z}_{1}}{2 c_{h} n_{1} \phi_{1} \bar{z}_{1}}\right)^{2}+\frac{\phi_{1} \bar{\ell}(1-\kappa)+\zeta c_{h}}{c_{h} n_{1} \phi_{1} \bar{z}_{1}}} \\
& =: K\left[n_{1}(t), n_{2}(t)\right] .
\end{aligned}
$$

\footnotetext{
${ }^{20}$ There exists a second solution of (38) which can be excluded since it implies a negative value of $\tau$.
} 